\title{
A CHARACTERIZATION OF HYPOELLIPTIC DIFFERENTIAL OPERATORS WITH VARIABLE COEFFICIENTS
}

\author{
R. E. WHITE ${ }^{1}$
}

ABSTRACT. Let $P$ be a linear differential operator with coefficients in $C^{\infty}(\Omega)$ where $\Omega \subset \mathrm{R}^{n}$. We characterize the hypoelliptic operators in terms of the $*$-hypoelliptic operators. $P$ is defined to be $*$-hypoelliptic on $\Omega$ if and only if $u \in \mathscr{D}_{F}^{\prime}(\Omega)$ and $P u \in C^{\infty}(\Omega)$ imply $u \in C^{\infty}(\Omega)$. We characterize the *-hypoelliptic operators via a priori estimates. We prove $P$ is hypoelliptic on $\boldsymbol{\Omega}$ if and only if for $u \in D^{\prime}(\boldsymbol{\Omega})$ and $P u \in C^{\infty}\left(\boldsymbol{\Omega}^{\prime}\right)$ with $\boldsymbol{\Omega}^{\prime} \subset \boldsymbol{\Omega}$, there exists for each $x_{0} \in \boldsymbol{\Omega}^{\prime}$ a relatively compact open neighborhood $\boldsymbol{\Omega}_{x_{0}} \subset \boldsymbol{\Omega}^{\prime}$ of $x_{0}$ such that $P$ is $*$-hypoelliptic on $\boldsymbol{\Omega}_{x_{0}}$.

Throughout this paper we will consider linear differential operators whose coefficients are in $C^{\infty}(\Omega)$ where $\Omega \subset \mathbf{R}^{n}$. Schwartz [8] first defined hypoelliptic operators on $\Omega \subset \mathbf{R}^{n}$. A differential operator $P$ is bypoelliptic on $\Omega \subset \mathbf{R}^{n}$ if and only if $u \in \mathscr{D}^{\prime}(\Omega)$ and $P u \in C^{\infty}\left(\Omega^{\prime}\right)$, where $\Omega^{\prime} \subset \Omega$, imply $u \in C^{\infty}\left(\Omega^{\prime}\right)$. Weyl [10] showed that weak solutions of Laplace's equation are in $C^{\infty}$. Hörmander [2] characterized the hypoelliptic operators with constant coefficients. Hörmander [3] and Malgrange [5] have shown that the formally hypoelliptic operators are hypoelliptic. This class includes the elliptic and $p$-parabolic operators with variable coefficients. Fedii. [1] gives sufficient conditions which allow us to conclude that

$$
P=-\frac{\partial^{2}}{\partial x^{2}}-\phi^{2}(x) \frac{\partial^{2}}{\partial y^{2}}
$$

is hypoelliptic on $\mathbf{R}^{2}$ where $\phi(x) \in C^{\infty}\left(\mathbf{R}^{2}\right), \phi(x)>0$ for $x \neq 0$ and $\phi(0)=$

Presented to the Society November 17, 1973; received by the editors October 23, 1973.

AMS (MOS) subject classifications (1970). Primary 35H05, 35D10; Secondary 46F05, 46A30.

Key words and phrases. Hypoelliptic on $\Omega, *$-hypoelliptic on $\Omega$, F réchet space, open mapping theorem, local on $\Omega$, *-local on $\Omega$, inductive limit.

1 This paper is part of a doctoral dissertation written under Professor Y. W. Chen at the University of Massachusetts in Amherst. 
0. In Theorem 6. we characterize the hypoelliptic operators with variable coefficients.

The condition of formal hypoellipticity and the sufficient conditions in Fedii [1] imply for all $x_{0} \in \Omega^{\prime}$ and for any $\Omega^{\prime} \subset \Omega$ there exists a relatively compact open neighborhood $\Omega_{x_{0}} \subset \Omega^{\prime}$ of $x_{0}$ such that for $u \in \mathscr{D}_{F}^{\prime}\left(\Omega_{x_{0}}\right)=$ $\bigcup_{m}\left(C_{c}^{m}\left(\Omega_{x_{0}}\right)\right)^{\prime}$ and $P u \in C^{\infty}\left(\Omega_{x_{0}}\right)$ imply $u \in C^{\infty}\left(\Omega_{x_{0}}\right)$. Operators with this property are hypoelliptic. Hence we define *-hypoellipticity on $\Omega$.

Definition. $P$ is *-bypoelliptic on $\Omega$ if and only if $u \in \mathscr{D}_{F}^{\prime}(\Omega)$ and $P u \in$ $C^{\infty}(\Omega)$ imply $u \in C^{\infty}(\Omega)$.

First, in Theorem 3, we characterize *-hypoelliptic operators on $\Omega$, and then in Theorem 6.we characterize hypoelliptic operators via *-hypoelliptic operators on neighborhoods of elements in $\Omega$. For the constant coefficient case, hypoellipticity is characterized algebraically in terms of the differential polynomial. The characterization of $*$-hypoelliptic operators will be in terms of a priori estimates one of which is similar to the subelliptic estimates for elliptic operators.

In order to characterize *-hypoelliptic operators on $\Omega$, we will need a theorem about linear operators on subspaces of $\mathscr{D}^{\prime}(\Omega)$. The motivation for this theorem comes from a discussion in Trèves [9, p. 538] of local fundamental solutions of an operator whose transpose is hypoelliptic.

Theorem 1. Let $E, F, G$ be Fréchet spaces such that $\mathcal{D}(\Omega) \subset E, F, G \subset$ $D^{\prime}(\Omega)$, the inclusion maps are continuous and the topologies are given by sequences of seminorms $\left(p_{n}\right),\left(q_{n}\right)$ and $\left(g_{n}\right)$ respectively. Also let $Q, P$ : $E \rightarrow D^{\prime}(\Omega)$ be continuous linear maps and define $R=\{u \in E \mid P u \in F\}$. If $Q: R \rightarrow G$ is a linear map, then $R$ may be made into the same Fréchet space by either of the sequences of seminorms

$$
\alpha_{n}(u) \equiv p_{n}(u)+q_{n}(P u)+g_{n}(Q u) \text { and } \gamma_{n}(u) \equiv p_{n}(u)+q_{n}(P u) \text {. }
$$

Proof. Since $P: E \rightarrow \mathscr{D}^{\prime}(\Omega)$ is linear, $R$ is a vector space. Since $Q$ : $R \rightarrow G$, both $\alpha_{n}$ and $\gamma_{n}$ are well defined. Since $P$ and $Q$ are linear maps, $\alpha_{n}$ and $\gamma_{n}$ are seminorms. If the graph $R \times P R$ is closed in $E \times F$, the locally convex metric space $\left(R,\left(\gamma_{n}\right)\right)$ will be complete and hence a Fréchet space. Let $u_{n} \in R, u_{n} \rightarrow u \in E$ and $P u_{n} \rightarrow v$ in $F$. Since $P: E \rightarrow \mathscr{D}^{\prime}(\Omega)$ is continuous, $P u_{n} \rightarrow P u$ in $D^{\prime}(\Omega)$. Since $P u_{n} \rightarrow v$ in $F, P u_{n} \rightarrow v$ in $\mathscr{D}^{\prime}(\Omega)$. Thus $P u=v \in F$ and $u \in R$.

In order to show $\left(R,\left(\alpha_{n}\right)\right)$ is complete, let $\left(u_{n}\right)$ be Cauchy in $\left(R,\left(\alpha_{n}\right)\right)$. Since $\left(R,\left(\gamma_{n}\right)\right)$ and $G$ are complete, there exist $u \in R$ and $v \in G$ such that 
$u_{n} \rightarrow u$ in $E, P u_{n} \rightarrow P u$ in $F$ and $Q u_{n} \rightarrow v$ in $G$. Since $Q$ is continuous on $E, Q u_{n} \rightarrow Q u$ in $\mathscr{D}^{\prime}(\Omega)$. Also $Q u_{n} \rightarrow v$ in $\mathscr{D}^{\prime}(\Omega)$. Thus $Q u=v \in G$ and $\left(R,\left(\alpha_{n}\right)\right)$ is a Fréchet space. Since the identity map from $\left(R,\left(\alpha_{n}\right)\right) \rightarrow$ $\left(R,\left(\gamma_{n}\right)\right)$ is continuous, we may apply the open mapping theorem in order to get our desired conclusion.

In the next theorem we establish necessary conditions for $*$-hypoellipticity. These conditions will be a priori estimates on subspaces of $H_{s}^{\text {loc }}(\Omega)$. Recall $H_{s}=\left\{u \in \mathcal{S}^{\prime} \mid \hat{u}(\xi)=\right.$ the Fourier transform of $u(x)$ is a function and $\left.\hat{u}(\xi)\left(1+|\xi|^{2}\right)^{s / 2} \in L_{2}\right\}$. With the norm $\|u\|_{s}=\left\|\hat{u}(\xi)\left(1+|\xi|^{2}\right)^{s / 2}\right\|_{L_{2}}, H_{s}$ is a Banach space. $H_{s}^{c}(K)=\left\{u \in H_{s}\right.$ |support $u \subset K, K$ compact $\}$ is a closed subspace of $H_{s}$ and hence a Banach space.

$$
H_{s}^{\text {loc }}(\Omega)=\left\{u \in \mathcal{D}^{\prime}(\Omega) \mid \phi u \in H_{s} \text { for all } \phi \in C_{c}^{\infty}(\Omega)\right\}
$$

is topologized via the sequence of seminorms $p_{n}(u)=\left\|\phi_{n} u\right\|_{s}$, where $\phi_{n} \epsilon$ $C_{c}^{\infty}(\Omega)$, support $\phi_{n} \subset$ support $\phi_{n+1}, \phi_{n+1} \equiv 1$ on support $\phi_{n}$ and $\bigcup_{n}$ support $\phi_{n}$ $=\Omega . H_{s}^{l o c}(\Omega)$ is complete and hence a Fréchet space. For the proofs of these facts see Hörmander [4]. Let

$$
P^{\alpha}(\xi)=\frac{\partial^{|a|}}{\partial \xi_{1}^{a_{1}} \cdots \partial \xi_{n}^{a_{n}}} P(\xi)=i^{|a|} D^{a} P(\xi)
$$

and

$$
[P, \phi] u=P(\phi u)-\phi P u=\sum_{0<|a|} \frac{D^{a} \phi}{\alpha !} \cdot P^{a} u
$$

Theorem 2. If $P$ is *-hypoelliptic on $\Omega$, then the following hold for all $s \in \mathbf{R}:$

(A) For all $\phi_{n}$ there exist $\phi_{m}$ and $C=C(n, s)>0$ such that

$$
\left\|\phi_{n} u\right\|_{s} \leq C\left(\left\|\phi_{m} P u\right\|_{m}+\left\|\phi_{m} u\right\|_{s-1}\right)
$$

where $u \in\left\{u \in H_{s-1}^{l o c}(\Omega) \mid P u \in C^{\infty}(\Omega)\right\}$. :

(B) For all compact $K \subset \Omega$ there exist $m$ and $C=C(K, s)>0$ such that

$$
\|u\|_{s} \leq C\left(\|P u\|_{m}+\|u\|_{s-1}\right)
$$

where $u \in\left\{u \in H_{s-1}^{c}(K) \mid P u \in C_{c}^{\infty}(K)\right\}$.

(C) For all $\phi_{n}$ and $\phi \in C_{c}^{\infty}(\Omega)$ there exist $\phi_{m}$ and $C=C(n, \phi, s)>0$ such that 


$$
\left\|\phi_{n}[P, \phi] u\right\|_{n} \leq C\left(\left\|\phi_{m} P u\right\|_{m}+\left\|\phi_{m} u\right\|_{s-1}\right)
$$

where $u \in\left\{u \in H_{s-1}^{\text {loc }}(\Omega) \mid P u \in C^{\infty}(\Omega)\right\}$.

Proof. In order to prove estimate $(\mathrm{A})$ apply Theorem 1 with $E=H_{s-1}^{\text {loc }}(\Omega)$, $F=C^{\infty}(\Omega), G=H_{s}^{\text {loc }}(\Omega), p_{n}(u)=\left\|\phi_{n} u\right\|_{s-1}, q_{n}(u)=\left\|\phi_{n} u\right\|_{n}, g_{n}(u)=\left\|\phi_{n} u\right\|_{s}$, $P=P$ and $Q$ the inclusion map $H_{s-1}^{\text {loc }}(\Omega) \rightarrow \mathscr{D}^{\prime}(\Omega)$. The assumption that $P$ is *-hypoelliptic on $\Omega$ and the fact that $H_{s-1}^{\text {loc }}(\Omega) \subset \mathscr{D}_{F}^{\prime}(\Omega)$ yields

$$
R=\left\{u \in H_{s-1}^{\text {loc }}(\Omega) \mid P u \in C^{\infty}(\Omega)\right\} \subset H_{s}^{\text {loc }}(\Omega)=G .
$$

From the conclusion of Theorem 1 we have for all $n$ there exist $m$ and a constant $C>0$ such that $\alpha_{n}(u) \leq C \gamma_{m}(u)$ for all $u \in R$. This gives our desired estimate.

Estimate (B) follows from estimate (A) for a suitable choice of $\phi_{n}$. This estimate may al so be proved by a direct application of Theorem 1 .

In order to prove estimate (C), let $E=H_{s-1}^{\text {loc }}(\Omega), F=C^{\infty}(\Omega), P=P$ and $Q$ the map from $H_{s-1}^{\text {loc }}(\Omega) \rightarrow \mathcal{D}^{\prime}(\Omega)$ given by $u \mapsto[P, \phi] u$. The assumption in Theorem 1 that $R=\left\{u \in H_{s-1}^{\text {loc }}(\Omega) \mid P u \in C^{\infty}(\Omega)\right\} \subset C^{\infty}(\Omega)=G$ follows from the *-hypoellipticity on $\Omega$. By the conclusion of Theorem 1 we obtain estimate (C).

Theorem 3. The following are equivalent:

1. $P$ is *-hypoelliptic on $\Omega$.

2. Estimate (A) holds for all $s \in \mathbf{R}$.

3. Estimates (B) and (C) hold for all $s \in \mathbf{R}$.

Proof. Theorem 2 gives $1 \Rightarrow 2$ and $1 \Rightarrow 3$. First we will show $2 \Rightarrow 1$. Let $u \in \mathscr{D}_{F}^{\prime}(\Omega)$ and $P u \in C^{\infty}(\Omega)$. Since $u \in \mathscr{D}_{F}^{\prime}(\Omega), u \in H_{s-1}^{\text {loc }}(\Omega)$ for some $s \in \mathbf{R}$. Apply estimate $(A)$ for this $s \in \mathbf{R}$. This gives for all $\phi_{n},\left\|\phi_{n} u\right\|_{s}<$ $\infty$ and hence $u \in H_{s}^{\text {loc }}(\Omega)$. Iterate this to get $u \in \bigcap_{s} H_{s}^{\text {loc }}(\Omega)=C^{\infty}(\Omega)$.

In order to prove $3 \Rightarrow 1$, let $u \in \mathscr{D}_{F}^{\prime}(\Omega)$ and $P u \in C^{\infty}(\Omega)$. Since $u \in$ $H_{s-1}^{\mathrm{loc}}(\Omega)$ for some $s \in \mathbf{R}$, estimate (C) implies for suitable choice of $\phi_{n}$ that $\|[P, \phi] u\|_{n}<\infty$. Since $[P, \phi] u=\phi P u-P(\phi u),\|P(\phi u)\|_{n}<\infty$ for large $n$. In order to obtain $\|\phi u\|_{s}<\infty$, use estimate (B) with $u$ replaced by $\phi u$. This holds for all $\phi \in C_{c}^{\infty}(\Omega)$ and hence $u \in H_{s}^{\text {loc }}(\Omega)$. Iterate this to get $u \in \bigcap_{s \in \mathbf{R}}$ $H_{s}^{\text {loc }}(\Omega)=C^{\infty}(\Omega)$.

If $P$ is an elliptic operator, then one may show $\|u\|_{s+m} \leq$ $C\left(\|P u\|_{s}+\|u\|_{s+m-1}\right)$, where $m=\operatorname{order} P, u \in C_{c}^{\infty}(K)$ with $K \subset \Omega$ compact. Such estimates are called subelliptic. :Estimate (B) is similar to subelliptic estimates and, in fact, subelliptic estimates may be extended from $C_{c}^{\infty}(K)$ to 
$\left\{u \in H_{s+m-1}^{c}(K) \mid P u \in H_{s}^{c}(K)\right\}$. See Nieto [6] or Peetre [7] for a proof of this. It is interesting to note that in order to establish *-hypoellipticity on $\Omega$ for elliptic operators, we need only the extended subelliptic estimate and not estimate (C). If $u \in\left\{u \in H_{s+m-1}^{\text {loc }}(\Omega) \mid P u \in H_{s}^{\text {loc }}(\Omega)\right\}$, then $[P, \phi] u \in H_{s}^{\text {loc }}(\Omega)$ since the order of $[P, \phi]$ is $m-1$. Since $[P, \phi] u=P(\phi u)-\phi P u, P(\phi u) \epsilon$ $H_{s}^{c}(K)$ where support $\phi \subset K$. Apply the extended subelliptic estimate to conclude $u \in H_{s+m}^{10 c}(\Omega)$. Iterate this to conclude that elliptic operators are *hypoelliptic.

Definition. $P$ is local on $\Omega$ if and only if $u \in \mathscr{D}^{\prime}(\Omega)$ and $P u \in C^{\infty}\left(\Omega^{\prime}\right)$ with $\Omega^{\prime} \subset \Omega$ imply $P(\phi u) \in C^{\infty}\left(\Omega^{\prime}\right)$ for all $\phi \in C_{c}^{\infty}\left(\Omega^{\prime}\right)$. $P$ is *-local on $\Omega$ if and only if $u \in \mathscr{D}_{F}^{\prime}(\Omega)$ and $P u \in C^{\infty}(\Omega)$ imply $P(\phi u) \in C^{\infty}(\Omega)$ for all $\phi$ $\epsilon C_{c}^{\infty}(\Omega)$.

If for all $\Omega^{\prime} \subset \Omega$ such that $u \in D^{\prime}(\Omega)$ and $P u \in C^{\infty}\left(\Omega^{\prime}\right)$ there exists for each $x_{0} \in \Omega^{\prime}$ a relatively compact open neighborhood $\Omega_{x_{0}} \subset \Omega^{\prime}$ of $x_{0}$ with $P$ *-local on $\Omega_{x_{0}}$, then $P$ is local on $\Omega$. In order to show this, let $\phi \in C_{c}^{\infty}\left(\Omega^{\prime}\right), u \in D^{\prime}(\Omega)$ and $P u \in C^{\infty}\left(\Omega^{\prime}\right)$. Let $\left(\psi_{i}\right)$ be a partition of unity subordinate to the finite subcover $\left(\Omega_{x_{i}}\right)_{i} \in$ support $\phi$ of support $\phi$ which is compact.

$$
P(\phi u)=P\left(\sum_{i=1}^{M<\infty} \psi_{i} \phi u\right)=\sum_{i=1}^{M<\infty} P\left(\psi_{i} \phi u\right) .
$$

Since $P$ is *-local on each $\Omega_{x_{0}}, P$ is *-local on $\Omega_{x_{i}}$. Since $\Omega_{x_{i}}$ are relatively compact, $u \in \mathscr{D}_{F}^{\prime}\left(\Omega_{x_{i}}\right)$ and $P u \in C^{\infty}\left(\Omega_{x_{i}}\right)$. Hence $P\left(\psi_{i} \phi u\right) \in C^{\infty}\left(\Omega_{x_{i}}\right)$ and $P(\phi u) \in C^{\infty}\left(\Omega^{\prime}\right)$. We next characterize the *-local operators.

Theorem 4. The following are equivalent:

1. $P$ is *-local on $\Omega$.

2. For all $s \in \mathbf{R}, \phi_{n} \in C_{c}^{\infty}(\Omega)$, and $\phi \in C_{c}^{\infty}(\Omega)$, there exist $\phi_{m}$ and $C=C\left(s, \phi_{n}, \phi\right)>0$ such that

$$
\left\|\phi_{n} P(\phi u)\right\|_{n} \leq C\left(\left\|\phi_{m} P u\right\|_{m}+\left\|\phi_{m} u\right\|_{s-1}\right)
$$

where $u \in\left\{u \in H_{s-1}^{\text {loc }}(\Omega) \mid P u \in C^{\infty}(\Omega)\right\}$.

3. For all $s \in \mathbf{R}, \phi_{n} \in C_{c}^{\infty}(\Omega)$, and $\phi \in C_{c}^{\infty}(\Omega)$, there exist $\phi_{m}$ and $C=C\left(s, \phi_{n}, \phi\right)>0$ such that

$$
\left\|\phi_{n}[P, \phi] u\right\|_{n} \leq C\left(\left\|\phi_{m} P u\right\|_{m}+\left\|\phi_{m} u\right\|_{s-1}\right)
$$

where $u \in\left\{u \in H_{s-1}^{10 c}(\Omega) \mid P u \in C^{\infty}(\Omega)\right\}$. 
Proof. $1 \Rightarrow 2$ is a consequence of Theorem 1. Let $E=H_{s-1}^{\text {loc }}(\Omega), F=$ $C^{\infty}(\Omega), G=C^{\infty}(\Omega), P=P$ and $Q$ the map given by $u \mapsto P(\phi u)$. The fact that $Q: R=\left\{u \in H_{s-1}^{1 \circ c}(\Omega) \mid P u \in C^{\infty}(\Omega)\right\} \rightarrow G$ follows from $P$ being *-local on $\Omega .2 \Rightarrow 1$ follows from the inequality in 2 and the fact that for large $n$, $\phi_{n} P(\phi u)=P(\phi u)$. : In order to show $1 \Leftrightarrow 3$, note by $P(\phi u)=\phi P u+[P, \phi] u, P$ is *-local on $\Omega$ if and only if $u \in \mathscr{D}_{F}^{\prime}(\Omega)$ and $P u \in C^{\infty}$ imply $[P, \phi] u \in$ $C^{\infty}(\Omega)$ for all $\phi \in C_{c}^{\infty}(\Omega)$. Apply Theorem 1 as above, except let $Q$ be given by the map $u \mapsto[P, \phi] u$.

The next theorem is concemed with extensions in some sense of certain distributions on $U \subset \Omega$ to distributions on $\Omega$. This theorem allows us to characterize both the hypoelliptic and local operators on $\Omega$.

Theorem 5. Let $U^{\prime} \subset U \subset \Omega$ be open subsets in $\mathbf{R}^{n}$ such that $\overline{U^{\prime}}$ is a compact subset of $U$. If $u \in \mathscr{D}_{F}^{\prime}(U)$, then there exists $v \in \mathscr{D}^{\prime}(\Omega)$ such that $\left.v\right|_{U^{\prime}}=\left.u\right|_{U^{\prime}}$.

Proof. Since $u \in D_{F}^{\prime}(U)$, there exists an integer $m$ such that $u \epsilon$ $\left(C_{c}^{m}(U)\right)^{\prime}$. Since the inclusion map $C_{c}^{m} \overline{\left(U^{\prime}\right)} \rightarrow C_{c}^{m}(U)$ is continuous, $u \mid \overline{U^{\prime}}$ is continuous on $C_{c}^{m} \overline{\left(U^{\prime}\right)}$. Thus there exists a constant $C>0$ such that

$$
\left|\left(\left.u\right|_{\overline{U^{\prime}}}, \phi\right)\right| \leq C \sup _{x} \sum_{|a| \leq m}\left|D^{a} \phi(x)\right| \text { for all } \phi \in C_{c}^{\infty}\left(\overline{U^{\prime}}\right) \text {. }
$$

Let $\psi_{a} \in C_{c}^{\infty}(\Omega)$ be such that $\psi_{a} \equiv C$ on $\overline{U^{\prime}}$ and $|\alpha| \leq m$. Define

$$
p(\phi)=\sup _{x} \sum_{|a| \leq m}\left|\psi_{\alpha}(x) D^{\alpha} \phi(x)\right| \text { where } \phi \in C_{c}^{\infty}(\Omega) .
$$

Since $\left(\psi_{a}\right)$ is a finite family, and hence locally finite on $\Omega, p(\phi)$ is a seminorm from the usual topology on $C_{c}^{\infty}(\Omega)$. See Hörmander $[4$, p. 6] for what is essentially the proof of this. Thus $\left|\left(u \mid \overline{U^{\prime}}, \phi\right)\right| \leq p(\phi)$ for all $\phi \in C_{c}^{\infty} \overline{\left(U^{\prime}\right)}$, which is a closed subspace of $C_{c}^{\infty}(\Omega)$. By the Hahn-Banach theorem there exists $v \in \mathscr{D}^{\prime}(\Omega)$ such that $v\left|\overline{U^{\prime}}=u\right| \overline{U^{\prime}}$.

Theorem 6. $P$ is hypoelliptic on $\Omega$ if and only if for all $\Omega^{\prime} \subset \Omega$ with $u \in \mathscr{D}^{\prime}(\Omega)$ and $P u \in C^{\infty}\left(\Omega^{\prime}\right)$ there exists for each $x_{0} \in \Omega^{\prime}$ a relatively compact open neighborhood $\Omega_{x_{0}} \subset \Omega^{\prime}$ of $x_{0}$ such that $P$ is *-bypeolliptic on $\Omega_{x_{0}}$

Proof. Let $P$ be hypoelliptic on $\Omega$. Let $\Omega^{\prime} \subset \Omega$ be any open subset. Choose $\Omega_{x_{0}} \subset \Omega^{\prime}$ to be any relatively compact open neighborhood of $x_{0}$. Assume $u \in \mathscr{D}_{F}^{\prime}\left(\Omega_{x_{0}}\right)$ and $P u \in C^{\infty}\left(\Omega_{x_{0}}\right)$. In order to show $u \in C^{\infty}\left(\Omega_{x_{0}}\right)$, 
we must find a neighborhood $\Omega_{y} \subset \Omega_{x_{0}}$ of each $y \in \Omega_{x_{0}}$ such that $u \epsilon$ $C^{\infty}\left(\Omega_{y}\right)$. Let $\Omega_{y}$ be any relatively compact neighborhood of $y \in \Omega_{x_{0}}$ such that $\overline{\Omega_{y}} \subset \Omega_{x_{0}}$. By Theorem 5 there exists $v \in D^{\prime}(\Omega)$ such that $\left.v\right|_{\frac{\boldsymbol{\Omega}_{y}}{\boldsymbol{口}_{y}}}=\left.u\right|_{\overline{\boldsymbol{\Omega}_{y}}}$. Since $P u \in C^{\infty}\left(\Omega_{x_{0}}\right), P u=P v \in C^{\infty}\left(\Omega_{y}\right)$. : Since $P$ is hypoelliptic on $\Omega$, $u=\left.v\right|_{\Omega_{y}} \in C^{\infty}\left(\Omega_{y}\right)$.

Suppose $P$ is *-hypoelliptic on each $\Omega_{x_{0}}$ and let $u \in \mathscr{D}^{\prime}(\Omega)$ and $P u \in C^{\infty}\left(\Omega^{\prime}\right)$. We must show $u \in C^{\infty}\left(\Omega_{x_{0}}\right)$ for all the given $\Omega_{x_{0}} \subset \Omega^{\prime}$. Since $\Omega_{x_{0}}$ is relatively compact, $u \in \mathscr{D}_{F}^{\prime}\left(\Omega_{x_{0}}\right)$. Also $P u \in C^{\infty}\left(\Omega_{x_{0}}\right)$, and since $P$ is *-hypoelliptic on $\Omega_{x_{0}}$, $u \in C^{\infty}\left(\Omega_{x_{0}}\right)$.

Theorem 7. $P$ is local on $\Omega$ if and only if for all $\Omega^{\prime} \subset \Omega$ with $u \in D^{\prime}(\Omega)$ and $P u \in C^{\infty}\left(\Omega^{\prime}\right)$ there exists for each $x_{0} \subset \Omega^{\prime}$ a relatively compact open neighborbood $\Omega_{x_{0}} \subset \Omega^{\prime}$ of $x_{0} \in \Omega^{\prime}$ such that $P$ is *-local on $\Omega_{x_{0}}$ :

Proof. Let $P$ be local on $\Omega$. Let $\Omega^{\prime} \subset \Omega$ be any open subset and choose $\Omega_{x_{0}} \subset \Omega^{\prime}$ to be any relatively compact open neighborhood of $x_{0} \in \Omega^{\prime}$. Assume $u \in \mathscr{D}_{F}^{\prime}\left(\Omega_{x_{0}}\right)$ and $P u \in C^{\infty}\left(\Omega_{x_{0}}\right)$. Choose $\left(\Omega_{y_{i}}\right)$ to be an open locally finite cover of $\Omega_{x_{0}}$ such that $\bar{\Omega}_{y_{i}} \subset \Omega_{x_{0}}:$ By Theorem 5 there exists $v \epsilon$ $D^{\prime}(\Omega)$ such that $\left.v\right|_{\overline{\Omega_{y_{i}}}}=\left.u\right|_{\Omega_{y_{i}}} \cdot$ : Since $P u=P v \in C^{\infty}\left(\Omega_{y_{i}}\right)$ and $P$ is local on $\Omega, P(\psi \phi u) \in C^{\infty}\left(\Omega_{y_{i}}\right)^{y_{i}}$, where $\psi \in C_{c}^{\infty}\left(\Omega_{y_{i}}\right)$. Let $\left(\psi_{i}\right)$ be a partition of unity subordinate to $\left(\Omega_{y_{i}}\right)$.

$$
P(\phi u)=P\left(\sum_{i=1}^{M<\infty} \psi_{i} \phi u\right)=\sum_{i=1}^{M<\infty} P\left(\psi_{i} \phi u\right) \in C^{\infty}\left(\Omega_{x_{0}}\right) .
$$

The proof of the converse is given after the definitions of local and *local operators on $\Omega$.

Theorem 7 gives an interpretation of estimates (C) in Theorem 2. Estimates (B) in Theorem 2 imply the existence of local fundamental solutions for the transpose of $P$. For the proof of this see Trèves [9]. In fact, it is easy to prove that hypoelliptic operators on $\Omega$ are characterized by the existence of local fundamental solutions, which may be extended continuou sly to $\mathcal{E}^{\prime}\left(\Omega_{x_{0}}\right)$, of the transpose of $P$ and by $P$ being local on $\Omega$.

\section{REFERENCES}

1. V. S. Fedii, On a criterion for hypoellipticity, Mat. Sb. 85 (127) (1971), 1848 = Math. USSR Sb. 14 (1971), 15-46. MR 44 \#367.

2. L. Hörmander, On the theory of general partial differential operators, Acta Math. 94 (1955), 161-248. MR 17, 853.

3. On interior regularity of the solutions of partial differential equations, Comm. Pure Appl. Math. 11 (1958), 197-218. MR 21 \#5064. 
4. L. Hörmander, Linear partial differential operators, Die Grundlehren der math. Wissenschaften, Band 116, Academic Press, New York; Springer-Verlag, Berlin, 1963. MR 28 \#4221.

5. B. Malgrange, Sur une classe d'opérateurs différentiels hypoelliptiques, Bull. Soc. Math. France 85 (1957), 283-306. MR 21 \#5063.

6. J. Nieto, Eine Charakterisierung der elliptischen Differentialoperatoren, Math. Ann. 141 (1960), 22-42. MR 22 \#6933.

7. J. Peetre, Elliptic partial differential equations of higher order, University of Maryland, Lecture Series, no. 40, College Park, Md., 1962.

8. L. Schwartz, Théorie des distribution. Tomes I, II, Actualités Sci. Indust., nos. 1091 1, 1122, Hermann, Paris, 1950, 1951. MR 12, 31; 833.

9. F. Treves, Topological vector spaces, distributions and kernels, Academic Press, New York, 1967. MR 37 \#726.

10. H. Weyl, The method of orthogonal projection in potential theory, Duke Math. J. 7 (1940), 411-444. MR 2, 202.

DEPARTMENT OF MATHEMATICS, NORTH CAROLINA STATE UNIVERSITY, RALEIGH, NORTH CAROLINA 27607 